\title{
Hematopoietic stem cells made BETter by inhibition
}

\author{
Ludovica Marando ${ }^{1,2,3}$ and Brian J. P. Huntly ${ }^{1,2,3}$ \\ ${ }^{1}$ Wellcome Trust-MRC Cambridge Stem Cell Institute; ${ }^{2}$ Department of Haematology, University of Cambridge and ${ }^{3}$ Cambridge Institute \\ for Medical Research, Cambridge Biomedical Campus, UK
}

E-mail:bjph2@cam.ac.uk

doi:10.3324/haematol.2018.193706

B romodomain and extra terminal (BET) proteins comprise the ubiquitously expressed $\mathrm{BRD} 2, \mathrm{BRD} 3, \mathrm{BRD} 4$ and the testes specific BRDT., ${ }^{1,2}$ These multipurpose proteins contain tandem $\mathrm{N}$-terminal bromodomains that bind acetylated lysine residues of histone (and non-histone) proteins and other protein modules, such as the extra terminal domain, and in some (BRD4, BRDT), a C-terminal domain. They also mediate a number of effects including transcriptional activation via recruitment of other partner proteins. ${ }^{3}$ Inhibitors of these proteins are emerging as exciting new therapies for the treatment of hematologic and solid malignancies, offering the possibility of specifically targeting epigenetic readers. We and others have already demonstrated the preclinical efficacy of BET inhibitors in acute myeloid leukemia (AML) ${ }^{46}$ while several other papers have documented similar efficacy in myeloma, ${ }^{7}$ non-Hodgkin lymphoma, ${ }^{8}$ and acute lymphoblastic leukemia. ${ }^{9}$ These observations have led to several clinical trials that are currently underway to confirm the efficacy of these drugs in AML and other malignancies. Even though the most mature trials have recently reported limited objective responses of monotherapy in heavily pre-treated AML, lymphoma and myeloma patients, ${ }^{10-12}$ early data suggest that combination therapies with other small molecules or more conventional cytotoxic agents might be particularly promising. ${ }^{1.15}$

BET proteins have multiple functions. Amongst these, and considered critical for the maintenance of malignant transcription, they are implicated in the regulation of large or "super" enhancers that control a number of critical genes, including oncogenes pivotal for the maintenance of leukemia, such as $B C L-2, I R F 8$, and $c-M Y C$. Their downregulation upon treatment with small molecule BET inhibitors at least partially explains the effects of BET inhibition observed in hematologic and solid malignancies., ${ }^{4,13,15,16}$

However, despite several ongoing clinical trials, we know surprisingly little about the consequences of disrupting BET protein function in normal tissues. In this issue of the Journal, Wroblewski et al. ${ }^{17}$ therefore, address a highly relevant topic and describe the effects of the prototypic BET inhibitor JQ1 on normal hematopoiesis.

Surprisingly, upon JQ1 treatment, Wroblewski et al. identify an increase in phenotypic HSC proliferation and mobilization in mice. In addition, these effects seem sustained, and functionally, in the setting of competitive transplantation, JQ1 treated HSC appear to contribute more to hematopoiesis in primary and, importantly, in secondary recipients with no evidence of exhaustion, albeit follow up was only for 12 months following transplantation. This increased proliferation does not enhance radiosensitivity. On the contrary, JQ1 treated mice show faster count recovery following sublethal irradiation compared to untreated controls. Although the authors have not studied potential underlying mechanisms, they postulate that the effects might be mediated by JQ1 dependant suppression of Myb, ${ }^{18}$ given the phenotypic similarity between JQ1 treatment and a mouse model of reduced Myb activity. ${ }^{19}$

Due to its pharmacokinetic properties, JQ1 does not lead to sustained target inhibition, and due to its limited efficacy in pre-clinical models, JO1 has never been tested in clinical trials. ${ }^{20}$ Therefore, whether JQ1 induced expansion of the normal HSC pool described here is a "class effect" shared by more potent $\mathrm{BET}$ inhibitors needs to be carefully addressed by further studies. This is further called into question by the toxicity reports from the clinical trials that have consistently reported hematologic toxicity, in particular, a dose-dependent, non-cumulative, reversible thrombocytopenia, ${ }^{10,11}$ and by opposing reports in the literature regarding the effects of BET inhibition on normal HSC. In a mouse model of controlled BRD4 inhibition, using an inducible transgenic shRNA, Brd4 silencing caused a significant reduction in Lineage- Sca-1+ ${ }^{+}$c-kit ${ }^{+}$hematopoietic stem cells 12 weeks after hematopoietic reconstitution. ${ }^{21}$ However, as RNAi would not only lead to almost complete loss of Brd4, but would also target the non-bromodomain dependent functions of the protein, differences between these models, where inhibitors would only intermittently target the bromodomains, might be expected.

Certainly the possibility that BET inhibitors, in addition to exerting antitumor effects, could enhance recovery of normal hematopoiesis, especially after combination chemotherapy, is intriguing and could open up new avenues for the use of BET inhibitors in clinical practice (Figure 1). Other areas of utility for BET inhibition, as suggested by Wroblewski et al. ${ }^{17}$ could include mobilization of peripheral blood (PB) HSC from donors who fail more standard approaches. However, validation of this study with orally available BET inhibitors and specific testing of these hypotheses will be necessary.

BET inhibitors might also have a role against acute graftversus-host disease (GvHD) and, in fact, were initially designed as immunosuppressives. Wroblewski et al. ${ }^{17}$ and others have shown that BET inhibitors impair T-cell function. Specifically, Wroblewski et al. ${ }^{17}$ find that JO1 treatment causes increased apoptosis in T cells, and this is associated with reduced expression of the antiapoptotic BCL-2 as measured by RT-qPCR. Others have demonstrated that treatment with I-BET151 results in a reduction in the secretion of IL-6, TNF- $\alpha$, and IL-12 by stimulated dendritic cells (DCs). ${ }^{22}$ Both $\mathrm{T}$ cells and DCs are important mediators of GvHD in recipients of allogeneic stem cell transplant, suggesting that BET inhibitors may serve as a prophylactic therapy against acute GvHD. Again, the results suggested by the pre-clinical models need to be validated in clinical trials and the benefits must be weighed against the risks of hematologic toxicity, particularly thrombocytopenia and neutropenia.

In conclusion, BRD4/BET inhibition is becoming a novel 


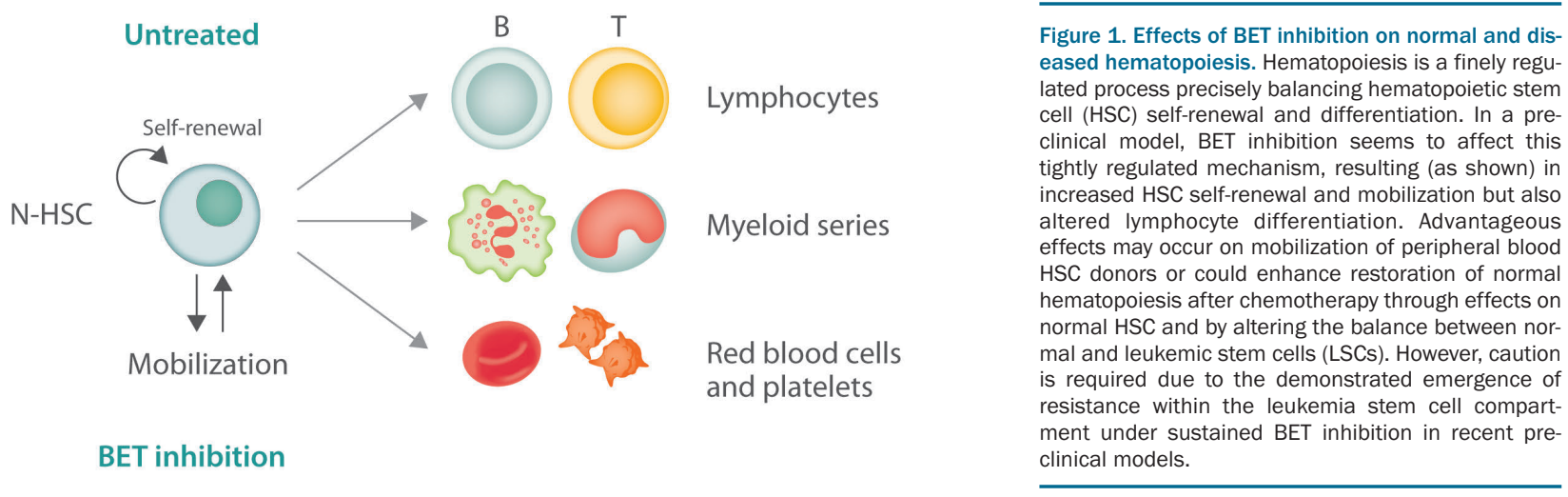

i. Homeostasis

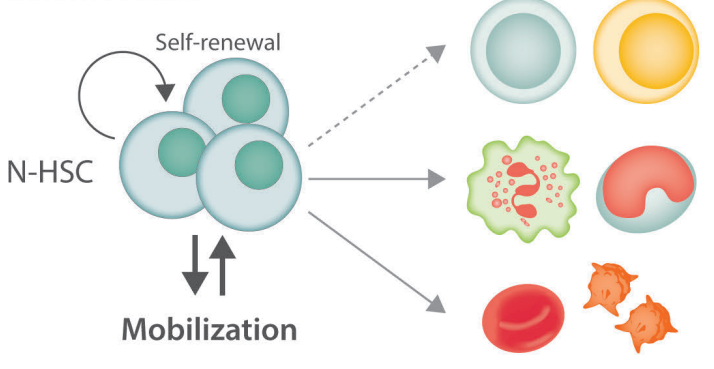

$\downarrow$ Lymphocytes

\author{
Use in marrow failure \\ + mobilization?
}

Red blood cells

and platelets

ii. Treatment of hematologic malignancies

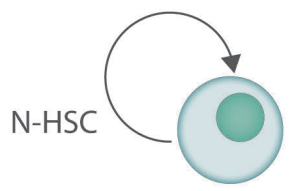

? Quicker regeneration

? Alteration of competition between HSC and LSC

? Effects on LSC

and exciting treatment option in many hematologic and solid malignancies, and may have novel promising effects in bone marrow transplantation. However, little is known about the effects of such treatment in normal tissues and an ongoing concern has been about the size of the therapeutic window. However, some reassurances are provided by the studies performed by Wroblewski et al. that have addressed this issue and suggest potentially beneficial effects on normal hematopoiesis; further studies are warranted to validate and extend these findings. However, a note of potential caution comes from models of BET inhibitor resistance that suggest that under the continuous and sustained selective pressures of BET inhibition, a small proportion of leukemic stem cells (LSC) survive and that over time these cells become the dominant clone. Mechanistically, this appears to occur through the activation of the ancillary WNT/ $\beta$-Catenin pathway that allows escape from BET inhibition. Importantly, resistance to BET inhibition in this model is only partially reversible on suspending I-BET. ${ }^{23}$ The evidence from the Fong et al. study, ${ }^{23}$ coupled with the evidence emerging from clinical trials and early study of combination therapy, strengthens the argument for developing rational combination strategies of BET inhibitors and other agents for patients with hematologic and solid malignancies. The Wroblewski et al. study ${ }^{17}$ promises an unexpected but welcome positive effect on normal hematopoiesis.

\section{References}

1 Wu SY, Chiang CM. The double bromodomain-containing chromatin adaptor Brd4 and transcriptional regulation. J Biol Chem. 2007;282 (18):13141-13145.

2. Pivot-Pajot C, Caron C, Govin J, Vion A, Rousseaux S, Khochbin S. Acetylation-dependent chromatin reorganization by BRDT: a testisspecific bromodomain-containing protein. Mol Cell Biol. 2003;23(15): 5354-5365.

3. Taverna SD, Li H, Ruthenburg AJ, Allis CD, Patel DJ. How chromatinbinding modules interpret histone modifica- tions: lessons from professional pocket pickers. Nat Struct Mol Biol. 2007;14(11):1025-1040.

4. Dawson MA, Gudgin EJ, Horton SJ, et al. Recurrent mutations: including NPM1c, activate a BRD4- dependent core transcriptional program in acute myeloid leukemia. Leukemia. 2014;28(2):311-320.

5 Dawson MA, Prinjha RK, Dittmann A, et al. Inhibition of BET recruitment to chromatine as an effective treatment for MLL fusion 
leukaemia. Nature. 2011;478(7370):529-533

6. Zuber J, Shi J, Wang E, et al. RNA1 screen identifies Brd4 as a therapeutic target in Acute Myeloid Leukaemia. Nature. 2011;478(7370):524 528.

7. Delmore JE, Issa GC, Lemieux ME, et al. BET bromodomain inhibition as a therapeutic strategy to target c-Myc. Cell. 2011;146(6):904-917.

8. Mertz JA, Conery AR, Bryant BM, et al. Targeting MYC dependence in cancer by inhibiting BET bromodomains. Proc Natl Acad Sci USA. 2011;108(40):16669-16674

9. Ott CJ, Kopp N, Bird L, et al. BET bromodomain inhibition targets both c-Myc and IL7R in high-risk acute lymphoblastic leukemia. Blood. 2012;120(14):2843-2852.

10. Berthon C, Raffoux E, Thomas X, et al. Bromodomain inhibitor OTX015 in patients with acute leukaemia: a dose-escalation, phase 1 study. Lancet Haematol. 2016;3(4):e186-195

11. Amorim S, Stathis A, Gleeson M, et al. Bromodomain inhibitor OTX015 in patients with lymphoma or multiple myeloma: a doseescalation, open-label, pharmacokinetic, phase 1 study. Lancet Haematol. 2016;3(4):e196-204.

12. Dawson M, Stein EM. Huntly BJP, et al. A Phase I Study of GSK525762, a Selective Bromodomain (BRD) and Extra Terminal Protein (BET) Inhibitor: Results from Part 1 of Phase I/II Open Label Single Agent Study in Patients with Acute Myeloid Leukemia (AML). Blood. 2017;130(Suppl 1):1377.

13. Fiskus W, Sharma S, Qi J, et al. Bet protein antagonist JQ1 is synergistically lethal with FLT3 tyrosine kinase inhibitor (TKI) and overcomes resistance to FLT3-TKI in AML cells expressing FLT-ITD. Mol Cancer Ther. 2014;13(10):2315-2327.
14. Fiskus W, Sharma S, Qi J, et al. Highly active combination of BRD4 antagonist and histone deacetylase inhibitor against human acute myelogenous leukemia cells. Mol Cancer Ther. 2014;13(5):1142-1154.

15. Herrmann H, Blatt K, Shi J, et al. Small-molecule inhibition of BRD4 as a new potent approach to eliminate leukemic stem- and progenitor cells in acute myeloid leukemia AML. Oncotarget. 2012;3(12):15881599.

16. Lovén J, Hoke HA, Lin CY, et al. Selective inhibition of tumor oncogenes by disruption of super-enhancers. Cell. 2013;153(2):320-334

17. Wroblewski M, Scheller-Wendorff M, Udonta F, et al. BET-inhibition by JQ1 promotes proliferation and self-renewal capacity of hematopoietic stem cells. Haematologica 2018;103(6):939-948.

18. Roe JS, Mercan F, Rivera K, Pappin DJ, Vakoc CR. BET Bromodomain Inhibition Suppresses the Function of Hematopoietic Transcription Factors in Acute Myeloid Leukemia. Mol Cell. 2015;58(6):1028-1039.

19. Sandberg ML, et al. c-Myb and p300 regulate hematopoietic stem cell proliferation and differentiation. Dev Cell. 2005;8(2):153-166.

20. Trabucco SE, Gerstein RM, Evens AM, et al. Inhibition of bromodomain proteins for the treatment of human diffuse large B-cell lymphoma. Clin Cancer Res. 2015;21(1):113-122.

21. Bolden JE, Tasdemir N, Dow LE, et al. Inducible in vivo silencing of Brd4 identifies potential toxicities of sustained BET protein inhibition. Cell Rep. 2014;8(6):1919-1929.

22. Sun Y, Wang Y, Toubai T, et al. BET bromodomain inhibition suppresses graft-versus-host disease after allogeneic bone marrow transplantation in mice. Blood. 2015;125(17):2724-2748.

23. Fong CY, Gilan O, Lam EY, et al. BET inhibitor resistance emerges from leukaemia stem cells. Nature. 2015;525(7570):538-542.

\section{NSG-S mice for acute myeloid leukemia, yes. For myelodysplastic syndrome, no.}

\section{Emmanuel Griessinger ${ }^{1}$ and Michael Andreeff ${ }^{, 3}$}

${ }^{1}$ INSERM U1065, Mediterranean Centre for Molecular Medicine (C3M), Team 4 Leukemia: Molecular Addictions, Resistances \& Leukemic Stem Cells, Nice, France; ${ }^{2}$ Faculté de Médecine, Université de Nice Sophia Antipolis, Nice, France and ${ }^{3}$ Section of Molecular Hematology and Therapy, Departments of Leukemia and Stem Cell Transplantation and Cellular Therapy, University of Texas MD Anderson Cancer Center, Houston, TX, USA

E-mail: emmanuel.griessinger@gmail.com or mandreef@mdanderson.org

doi:10.3324/haematol.2018.193847

$\mathrm{R}$ esearch on primary patient cells is a compelling challenge for scientists. Although initially limited to short experiments over hours or days, engrafting these primary human cells in immunodeficient mice today allows even more informative investigation to be carried out over weeks and months. This experiment is fascinating, probably first because it gives rise to personal and moral questions about the patient's avatar. Also, in basic research, the xenograft is the model to be used to reveal the stemness properties of a certain population of cancer cells. ${ }^{1}$ Although today there are some ex vivo alternatives, the xenograft remains the gold standard technique to study cancer stem cells which are responsible for cancer initiation propagation, maintenance and evolution. Uncovering the presence of primary human leukemic cells in a sample of mouse tissue 10-16 weeks after injection, demonstrating the initial engraftment of leukemia initiating cells (LICs) causes an exhilarating sensation known to only a few lucky scientists. Absence of graft triggers the opposite sensation of complete disappointment, which has led several teams to focus their attention on this particular problem with the xenograft approach. In this issue of Haematologica, Krevvata et al. put forward fundamental new insights to help improve xenograft of acute myeloid leukemia (AML) and myelodysplastic syndrome (MDS). ${ }^{2}$

Myelodysplastic syndrome and acute myeloid leukemia are myeloid neoplasms that disrupt normal hematopoiesis. This group of myeloid leukemias could be considered as a continuum consisting of a multitude of different leukemias, including all possible myeloid abnormalities. This results in a wide range of severity and patient overall survival (OS). MDS patients have globally better OS than AML patients, and some MDS evolve inevitably towards AML. Interestingly, the first attempts at AML/MDS xenograft quickly revealed, through the repartition of samples engrafting and non-engrafting the mice, that the engraftment potential was perfectly linked with the aggressiveness of the leukemia, since AML samples are usually more easy to engraft than MDSs. ${ }^{3,4}$ Many independent studies have offered different reasons for engraftment failure, but none can satisfactorily explain it. Possible explanations are either related to the host immune environment or to the defect of the grafted cells or to the graft and host compatibility.

The innate and adaptive immune response of the host environment is an obvious and very clear obstacle for the 